\title{
Étudier la structure des membranes biologiques : l'intérêt des systèmes modèles et des neutrons
}

Giovanna Fragneto (fragneto@ill.eu)

Institut Laue-Langevin, 71 avenue des Martyrs, 38042 Grenoble Cedex 9

Les cellules, unités de base

des êtres vivants, sont bien

délimitées et séparées de

l'extérieur par des membranes.

Capables à la fois d'enfermer

les constituants cellulaires

et d'autoriser des échanges

avec l'extérieur, ces membranes

sont ultrafines. L'ensemble

des membranes d'un corps

humain couvre une superficie

de quelques hectares, mais

ne constitue qu'une petite

partie de notre masse.

Pour étudier la dynamique

et la fonction de ces objets

étonnants, les physiciens

cherchent en premier lieu

à comprendre leur structure.

Cela passe par des expériences

sur des systèmes modèles,

plus simples et mieux contrôlés,

grâce à une sonde de la matière

qui accède à différentes

échelles de taille et de temps :

les neutrons thermiques.
La cellule est une unité structurale et fonctionnelle de la plupart des organismes vivants ; un être humain en contient environ cent mille milliards. Chaque cellule est entourée d'une membrane. Une estimation sommaire indique que l'ensemble des membranes cellulaires de notre corps couvre une superficie d'environ trois hectares [1]. La membrane entourant la cellule est un élément essentiel dans tout être vivant. Elle doit à la fois être assez étanche pour séparer la cellule de l'extérieur et permettre les échanges de matière depuis et vers la cellule : nutrition, respiration. Elle est semi perméable et contribue à réguler activement ces échanges. Elle joue également un rôle dans la communication entre deux cellules voisines (transmission de signaux) et dans l'adhésion cellulaire. On trouve aussi une importante quantité de membranes à l'intérieur même d'une cellule, où elles délimitent les compartiments intracellulaires.

De nombreuses maladies sont liées à un problème de régulation de la composition membranaire : c'est le cas de l'athérosclérose. De même, le cholestérol est indispensable à l'activité cellulaire, et des mécanismes de transport inappropriés peuvent conduire à l'apparition de maladies mortelles.

\section{Pourquoi s'intéresser}

\section{à la structure des membranes biologiques?}

Les membranes sont fines, tout en étant robustes. Ces propriétés remarquables sont réalisées grâce à une structure optimisée par des milliards d'années d'évolution.
Les principaux composants structurels des membranes sont des lipides, en majorité des phospholipides, mais aussi du cholestérol. Les phospholipides sont des molécules qui ont une partie (tête) qui aime l'eau, hydrophile, et une partie carbonée, hydrophobe. En présence d'eau, ils sont capables de s'auto-assembler sous forme de bicouche continue (fig. 1) : ils présentent leurs têtes vers l'extérieur, donc vers les milieux aqueux à l'intérieur et à l'extérieur de la cellule. Les parties carbonées sont prises en sandwich et forment une barrière.

Les membranes peuvent incorporer une grande quantité de protéines variées, dites "membranaires ", pouvant constituer jusqu'à la moitié de leur masse, et parfois plus (fig. 1). Ces protéines s'intègrent entre les queues des lipides et participent aux échanges de matériel d'un côté à l'autre de la membrane.

Très robustes, d'une épaisseur d'environ $5 \mathrm{~nm}$ pour une surface de plusieurs dizaines de micromètres carrés, les bicouches peuvent servir de support expérimental aux études théoriques d'objets à deux dimensions. Ainsi, des modèles de la forme des bicouches ont été élaborés, basés sur leur tension et leur rigidité de courbure. Ces mêmes modèles permettent de comprendre comment des objets fins, comme les membranes, fluctuent sous l'effet de leurs interactions avec les molécules d'eau : la rigidité de courbure contrôle les toutes petites échelles; les grandes échelles sont contrôlées par une éventuelle force extérieure (par exemple la présence d'une paroi solide ou d'une cellule voisine) ; entre les deux, les échelles intermédiaires sont régies par la tension de surface. 


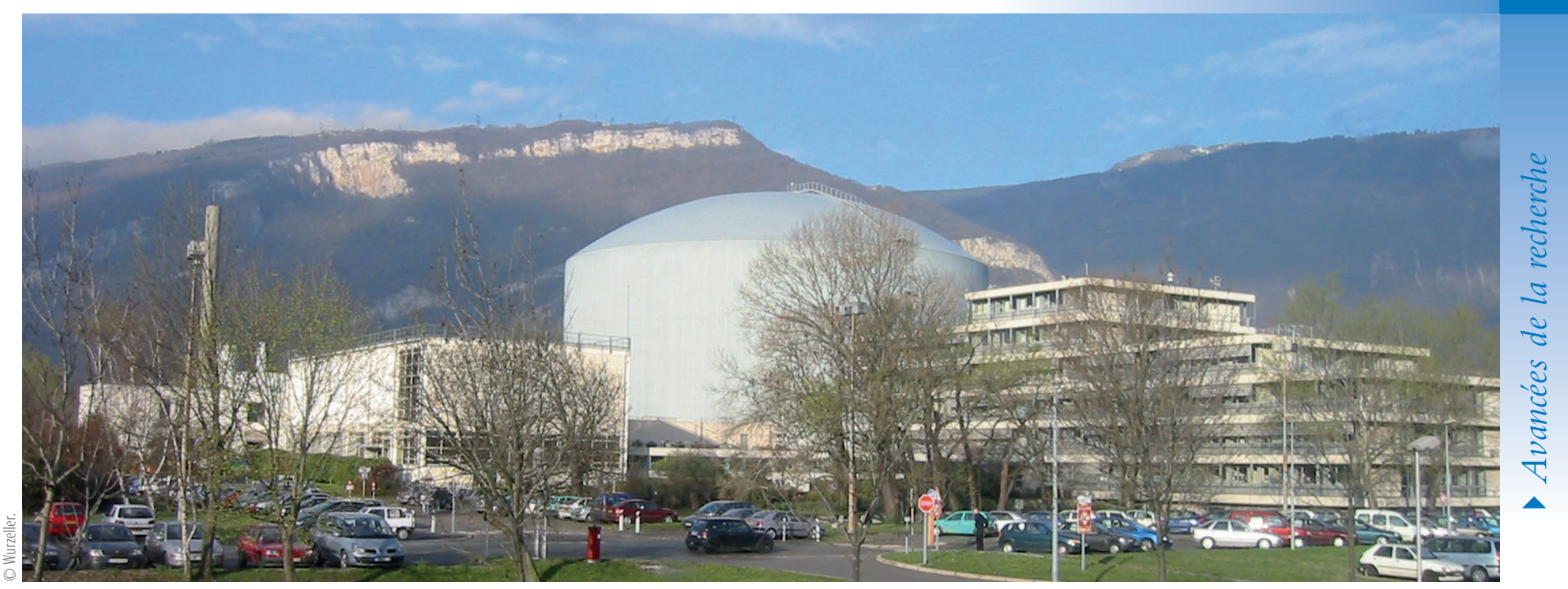

L'Institut Laue-Langevin à Grenoble est un centre de recherche international. Le réacteur, modéré à l'eau lourde, d'une puissance de 58 MW, source intense de neutrons thermiques, se trouve sous la coupole. Une quarantaine d'instruments scientifiques, placés tout autour du cœur du réacteur, permettent des applications allant de la physique fondamentale à la biologie, en passant par la cristallographie, la chimie ou la science des matériaux.

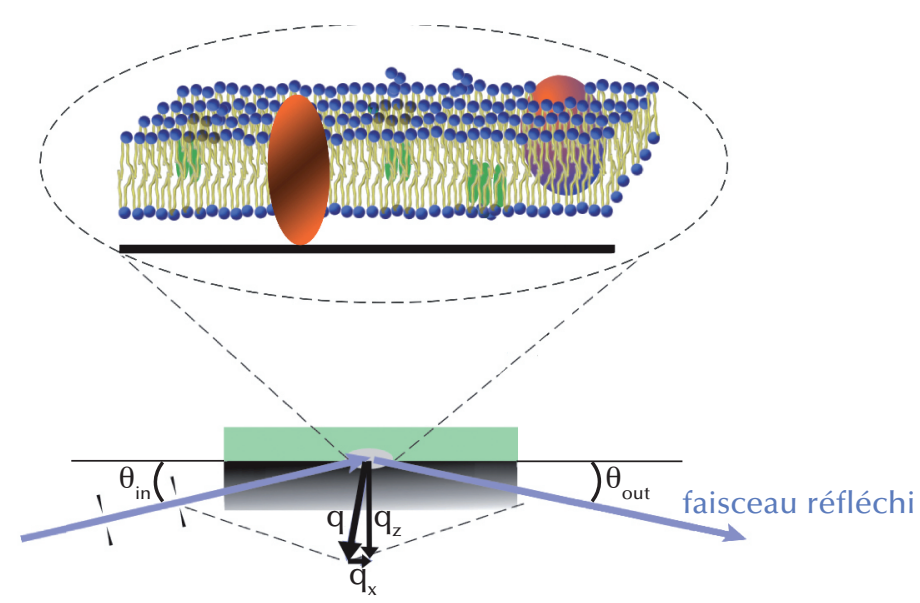

1. Bicouche de lipides sondée par réflectivité de neutrons.

En haut : schéma d'une bicouche de lipides, ici déposée sur une surface solide (en noir). Les phosphocholines sont des lipides constitués d'une extrémité qui se place dans l'eau (tête « hydrophile », schématisée par une boule bleue) et de deux longues chaînes carbonées insolubles dans l'eau (queues " hydrophobes ", dans lesquelles les groupes carbone-hydrogène sont représentés en jaune). Elles se disposent en bicouches : deux feuillets, où les têtes se placent face à l'eau. Des protéines, dites « membranaires » (en vert et orange), peuvent s'insérer dans une bicouche. En bas : en réflectivité de neutrons, un faisceau délimité par des fentes arrive sur la surface avec un angle d'incidence $\theta_{\text {in }}$. L'analyse du faisceau réfléchi (réflectivité spéculaire, $\theta_{\text {out }}=\theta_{\text {in }}$, comme sur un miroir) permet d'extraire des informations sur la structure et la composition des lipides dans la direction perpendiculaire au plan de la bicouche $\left(q_{z}\right)$. En plaçant le détecteur hors du faisceau réfléchi (réflectivité non spéculaire, $\theta_{\text {out }} \neq \theta_{\text {in }}$ ), on sonde la structure de la bicouche dans son plan $\left(q_{x}\right)$.

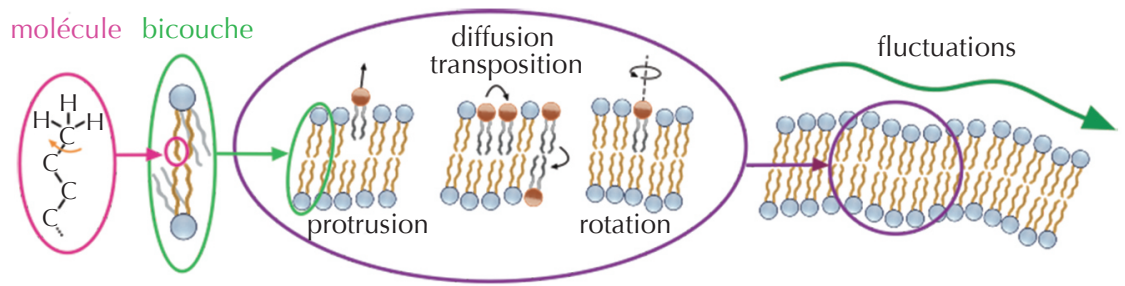

\begin{tabular}{lll}
$\sim 6 \times 10^{2} \mathrm{~nm}^{-1}$ & Vecteur de diffusion & $\sim 10^{-2} \mathrm{~nm}^{-1}$ \\
\hline$\sim 0,01 \mathrm{~nm}$ & Échelle de longueur & $\sim 6 \times 10^{2} \mathrm{~nm}$ \\
\hline$\sim 10^{3} \mathrm{meV}$ & Transfert d'énergie & $\sim 10^{-6} \mathrm{meV}$ \\
\hline$\sim 6 \times 10^{-3} \mathrm{ps}$ & Échelle de temps & $\sim 6 \times 10^{6} \mathrm{ps}$ \\
\hline
\end{tabular}

2. Échelles de taille, de temps et d'énergie caractéristiques de la structure et de la dynamique des bicouches, accessibles aux neutrons.
Les lipides des membranes soulèvent des questions de fond. Par exemple, pourquoi y a-t-il dans les membranes des différentes cellules une grande variété de lipides, au moins 1500 types différents ? Et pourquoi tous ces lipides sont-ils chimiquement différents de ceux des anciennes bactéries, les Archaea ? Pourquoi est-ce que l'on observe à l'état naturel une ségrégation des lipides et non pas un mélange complet ? Plus précisément, il a été observé des structures qui représentent entre 10\% et $30 \%$ du volume de la membrane. Elles sont surnommées "radeaux " car ce sont des microdomaines rigides qui se déplacent latéralement en bloc, comme s'ils flottaient, dans le plan de la membrane. Quels sont leurs propriétés et leur intérêt pour la cellule?

Les études de bicouches lipidiques sont importantes, tant du point de vue fondamental qu'en raison de la grande variété des applications possibles. Elles ont un intérêt pharmacologique, puisque le transport d'un médicament à travers une membrane dépend des propriétés physico-chimiques de celle-ci. Leurs applications en nanobiotechnologie incluent le développement de biocapteurs. Les revêtements biofonctionnels des organes artificiels et les dispositifs médicaux implantés sont d'autres domaines d'utilisation.

La robustesse des bicouches est compatible avec leur grande adaptabilité, liée à la richesse de leur dynamique. Ainsi, les molécules de lipides ont plusieurs types de mouvements individuels (fig. 2) [2] :

- D'une part des mouvements de diffusion au sein du plan de la membrane. Une \> 
membrane peut se comporter comme un solide à deux dimensions, avec les propriétés d'un gel et des coefficients de diffusion latérale des molécules relativement lents, inférieurs à $10^{-3}$ micromètres carrés par seconde. Elle peut subir une transition de phase (fig. 5) : au-dessus d'une température de transition, elle se comporte comme un liquide à deux dimensions. Les molécules y diffusent latéralement, comme si elles étaient libres : elles peuvent explorer en quelques secondes l'ensemble d'une membrane de plusieurs dizaines de micromètres carrés. Quand la membrane est composée d'un mélange de nombreux lipides différents, elle a en général ce comportement liquide.

- En outre, les lipides peuvent aussi facilement tourner sur eux-mêmes dans la membrane, en des temps rapides, de l'ordre de 10 nanosecondes.

- Ensuite, au sein de la bicouche, une molécule de lipide peut passer d'une monocouche de la membrane à une autre. Ce phénomène de transposition, ou flip-flop, peut prendre des minutes ou des heures (ce qui est très lent pour des molécules), car la structure de la bicouche est robuste : c'est coûteux en énergie de faire passer une tête polaire à travers la zone hydrophobe de la bicouche. Le flip-flop est favorisé par certaines molécules, telles que des enzymes, ou la présence de pores à travers la membrane.

- Enfin, un lipide peut sortir de la membrane sur une hauteur de l'ordre de la fraction de nanomètre. Ce phénomène de protrusion se produit sur une échelle de temps de l'ordre de la nanoseconde.

\section{Quels systèmes modèles?}

En raison de la complexité des membranes cellulaires, et de la variété des échelles de taille et de temps qu'elles mettent en jeu, leur caractérisation structurale est un défi. Une approche classique consiste à recourir à des systèmes simplifiés. Ceux-ci doivent être suffisamment proches des membranes réelles pour être qualifiés de biomimétiques, tout en étant assez simples pour être accessibles à des mesures physiques. Comme les membranes réelles, ils doivent être à la fois robustes et flexibles. Dans la plupart des cas, la composition est d'abord limitée à un seul type de lipide, avant de progressivement en mélanger plusieurs.
Historiquement, les empilements multilamellaires faits de centaines ou de milliers de bicouches ont été utilisés en premier. On peut définir et mesurer précisément leur hydratation. Déposés sur des surfaces solides, leur emplacement est bien contrôlé, ce qui permet de les positionner dans un faisceau de neutrons ou de rayons $\mathrm{X}$, par exemple. La grande quantité de matière qu'ils contiennent les rend accessibles même aux techniques peu sensibles.

Quand on s'intéresse aux effets de paramètres extérieurs comme le $\mathrm{pH}$ et la force ionique, ou aux interactions avec des molécules externes à la membrane, il est préférable d'analyser une seule bicouche à la fois, dans l'eau. Une bicouche supportée est déposée sur une paroi solide, qui l'attire et la maintient en place (fig. 1) [3]. En plaçant à quelques nanomètres de distance une bicouche flottante, c'est-à-dire une deuxième bicouche qui est libre (fig. 4), on peut étudier les fluctuations d'une bicouche, ou les interactions entre deux bicouches voisines [4].

Les vésicules (ou liposomes) sont des bicouches plus ou moins sphériques, qui enferment un volume d'eau (fig. 3). On peut fabriquer des petites vésicules en les forçant à passer à travers un filtre, avec des pores typiquement de quelques dizaines ou centaines de nanomètres de diamètre.

\section{Quelles études avec les neutrons?}

Aux échelles micrométriques, des techniques variées permettent de sonder la structure et les propriétés élastiques des bicouches lipidiques; c'est le cas de la microscopie optique ou de la diffusion de la lumière. Aux échelles bien plus petites, au niveau moléculaire, on utilise entre autres la résonance magnétique nucléaire, ou des simulations numériques qui élucident en détail les relations entre la structure chimique et les propriétés physiques de divers lipides. Entre ces échelles de la molécule et du micromètre, les techniques de diffusion de neutrons thermiques (d'énergie comprise entre $10^{-3}$ et $10^{-1} \mathrm{eV}$ ) jouent un rôle privilégié. Ce sont des outils non destructifs pour caractériser la structure et la dynamique des systèmes biomimétiques, aux échelles allant des dixièmes jusqu'aux dizaines de nanomètres et de la picoseconde à la milliseconde (fig. 2). Leur puissance tient surtout à leur pouvoir de pénétration. Du fait que leur charge électrique est nulle, les neutrons peuvent pénétrer la matière sans être arrêtés par une barrière coulombienne. Ils sondent ainsi l'intérieur des échantillons. Dans la matière non magnétique (comme ici l'eau et les macromolécules biologiques), leur interaction se fait essentiellement avec les noyaux atomiques, via les forces nucléaires à courte portée.

La principale originalité des techniques de diffusion neutronique est la possibilité d'utiliser la substitution isotopique pour mettre en évidence les parties d'intérêt dans les systèmes étudiés. L'hydrogène et son isotope le deutérium ont des propriétés chimiques similaires, mais ils interagissent de manière très différente avec les neutrons. Autrement dit, si l'on remplace certains noyaux d'hydrogène d'un échantillon de matière molle ou de biologie (ou ceux de l'eau environnante) par des noyaux de deutérium, on ne change quasiment pas les caractéristiques physiques et chimiques de l'échantillon, mais on annule le contraste aux neutrons de telle ou telle partie de l'échantillon, ce qui rend les autres sélectivement visibles [5].

Les techniques neutroniques qui ont été principalement utilisées pour les études des membranes donnent accès à des informations à des échelles variées (fig. 2). Ainsi :

- la diffraction détermine la structure des systèmes lamellaires lipidiques empilés ;

- la diffusion aux petits angles fournit des informations (moyennes, plutôt que détaillées) sur la forme, la taille et les interactions de systèmes en solution, ou des vésicules lamellaires lipidiques ;

- la réflectivité spéculaire (fig. 1) sonde la structure d'une bicouche dans la direction perpendiculaire à son plan ;

- la réflectivité non spéculaire et la diffusion aux petits angles en incidence rasante peuvent, entre autres, sonder les structures latérales aux échelles du micromètre ou du nanomètre, respectivement ;

- enfin, la diffusion inélastique permet d'étudier la dynamique, sur des échelles de longueur de un à cent nanomètres et des échelles de temps allant de la fraction de picoseconde à la milliseconde.

Par exemple, des expériences de réflectivité de neutrons ont permis de confirmer la présence de protrusions pour des lipides décorés avec le polymère soluble polyéthylène-glycol, ou de mesurer le potentiel d'interaction d'une bicouche de phosphocholines avec une paroi solide. 


\section{Vers des systèmes de plus en plus complexes}

Les membranes biologiques réelles ont différents composants, lipides et protéines, qui sont distribués de façon très hétérogène, formant des domaines ; ceux-ci sont impliqués dans la transmission de signaux entre l'intérieur et l'extérieur de la cellule. Les efforts actuels sont consacrés à l'exploration de systèmes, tels que des lipides naturels extraits de cellules, permettant de se rapprocher de plus en plus de cette réalité complexe.

Par exemple, le cholestérol est un composant de base des membranes. Il forme des domaines, donc sa distribution latérale est hétérogène. Il est présent en quantités différentes entre les deux feuillets de la membrane, ce qui crée également une hétérogénéité transversale : la monocouche interne à la cellule et la monocouche externe sont différentes. Nous avons étudié de telles bicouches asymétriques, en l'occurrence des bicouches flottantes fluides, où du cholestérol a été mélangé à des phospholipides en proportion réaliste (18\% molaire). Par réflectivité de neutrons, nous avons observé que le cholestérol ne migre pas d'une bicouche à l'autre [6].

En revanche, nous avons pu suivre la redistribution des phospholipides d'une bicouche à l'autre. Pour déterminer plus précisément à quelle vitesse se fait cette redistribution, nous avons eu recours à la deutération (fig. 3) : une grande quantité de vésicules faites de lipides contenant du deutérium ("vésicules deutérées ») est mise en présence d'une bicouche dont les lipides sont normaux ("bicouche hydrogénée "), ou vice versa. Grâce à la résolution spatiale et temporelle de la réflectivité des neutrons, il a été possible de suivre en fonction du temps l'échange des phospholipides grâce auquel, après trois heures, la bicouche finit par être complètement deutérée [7].

Nous avons aussi étudié le couplage du cholestérol à une molécule appelée le ganglioside GM1 [6]. Le GM1 s'associe au cholestérol pour former des domaines spécifiques, essentiels pour la cellule, non seulement pour la forme de la membrane (par exemple, ces domaines sont la base de la déformation en trois dimensions qui donne lieu à la formation de petites cavités, les « cavéoles "), mais aussi pour des fonctions biologiques telles que la régulation des

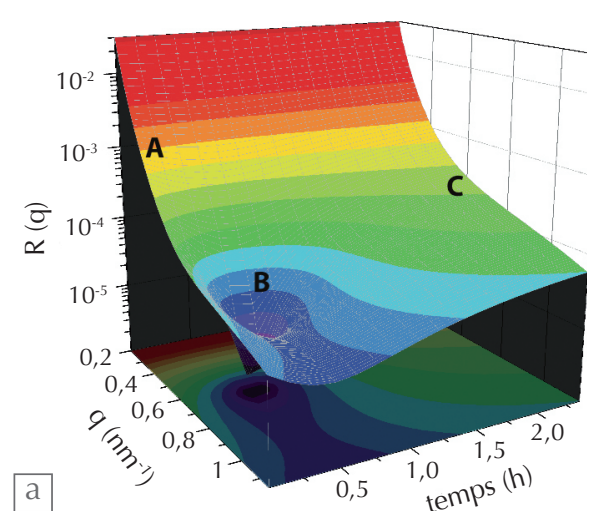

3. Échange de lipides entre bicouches. Une bicouche formée de lipides normaux (non deutérés), déposée sur une paroi solide (silicium), est mise en présence d'une suspension de vésicules faites de lipides deutérés.

(a) Réflectivité de neutrons. Le nombre de neutrons réfléchis, $R(q)$, est tracé en fonction du vecteur d'onde transféré, q. La mesure est répétée pendant près de trois heures, pour suivre la cinétique de l'échange.

(b) Interprétation des mesures (les lettres A, B et C renvoient à la figure (a)). Dans la bicouche (exagérément grossie par rapport aux vésicules), les lipides hydrogénés (jaunes) sont remplacés par les lipides deutérés (rouges) venant des vésicules en solution d'eau (normale, non deutérée). Le mécanisme de remplacement dans les deux feuillets (B) domine ici le mécanisme par insertion dans le premier feuillet, suivie de transposition. Le silicium, la bicouche et la solution ont des contrastes visibles aux neutrons, et les vésicules sont trop éloignées de la surface pour être visibles. En quelques dizaines de minutes, des lipides hydrogénés et deutérés sont mélangés, ce qui fait chuter le contraste (B). Après trois heures, tous les lipides de la bicouche sont deutérés (C), ce qui fait remonter le contraste avec la solution, donc R(q). (D’après [7].)

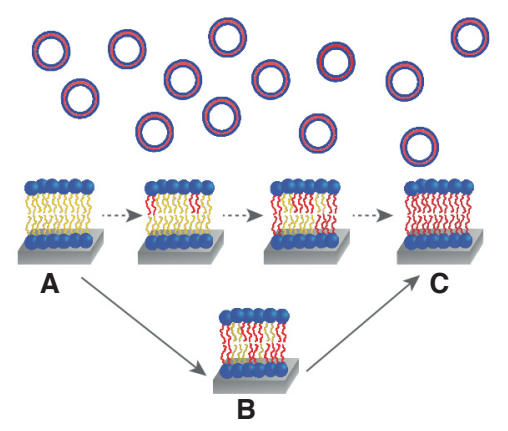

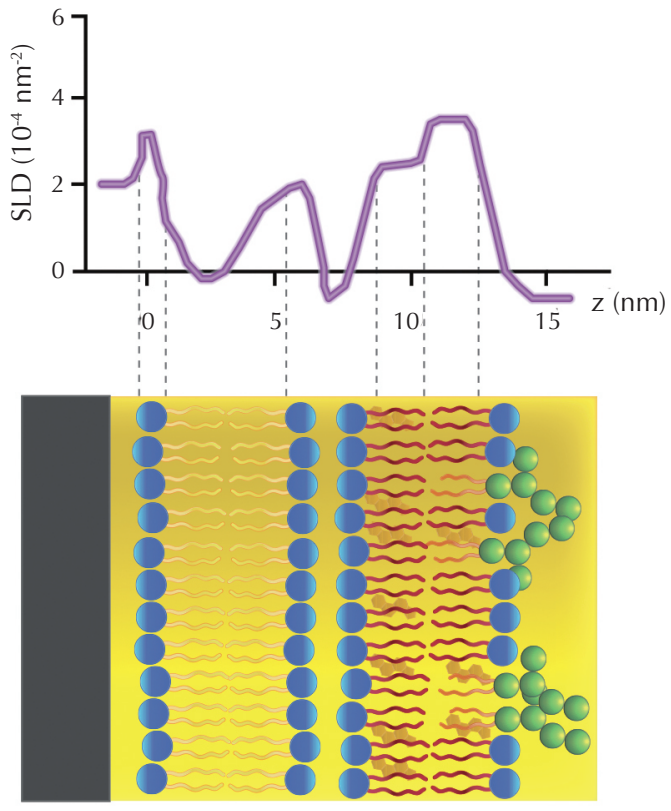

4. Cholestérol et ganglioside GM1 dans une bicouche flottante.

En haut : densité de longueur de diffusion (SLD) de neutrons en fonction de la distance z à la surface solide. La longueur de diffusion représente l'amplitude de l'onde diffusée par le noyau de l'isotope considéré. Dans ces unités, la matière hydrogénée apparaît proche de 0 , la matière deutérée proche de 6 , ce qui détermine le contraste. Entre $z=8,5$ et $12 \mathrm{~nm}$ (bicouche flottante), le profil est asymétrique.

En bas : Interprétation des mesures de réflectivité de neutrons. L'eau est représentée en jaune. La bicouche proche du substrat a des queues hydrogénées, représentées elles aussi en jaune pour indiquer qu’elles sont masquées par leur absence de contraste avec l'eau. La bicouche flottante a des queues deutérées (rouge), ce qui rend visible le cholestérol hydrogéné (beige) inséré entre les queues. Le ganglioside GM1 (têtes en vert) est inséré uniquement dans le deuxième feuillet de la bicouche flottante, et il régule la répartition de cholestérol à des valeurs d'environ $70 \%$ et 30\% dans le premier et le second feuillet respectivement. (D’après [6].) 

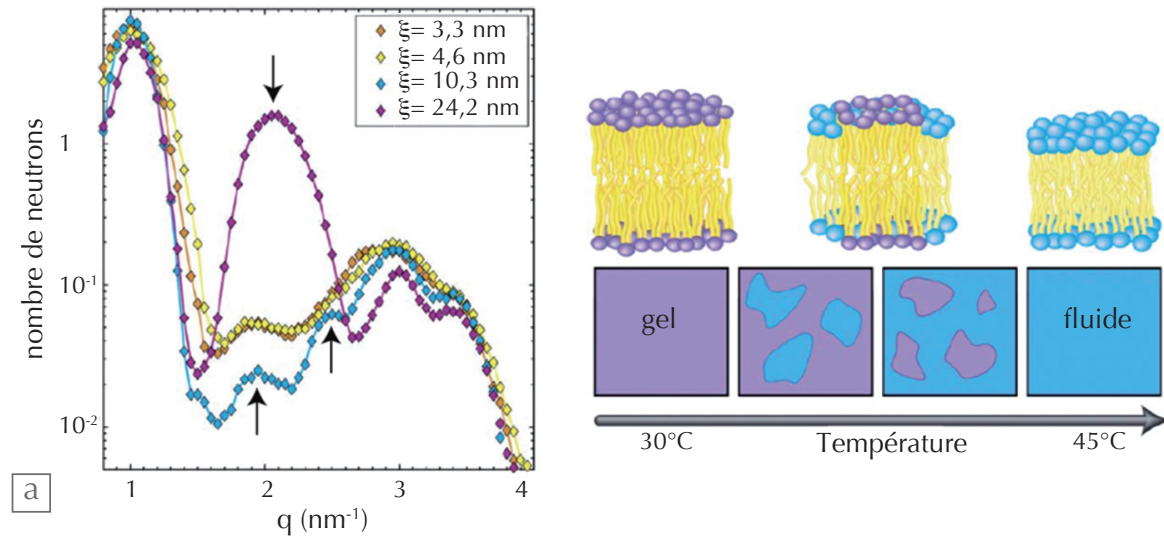

5. Transition gel-liquide à deux dimensions dans une bicouche faite d'un seul type de phospholipides, où les queues ont seize carbones.

(a) Diffraction de neutrons par des multicouches. Le nombre de neutrons diffractés, rapporté au nombre de neutrons incidents, est tracé en fonction du vecteur d'onde transféré q (grandeur qui dépend de l'angle $\theta$ et de la longueur d'onde $\lambda$ du faisceau incident : $q=4 \pi \sin \theta / \lambda$ ). Les courbes sont mesurées à $40^{\circ} \mathrm{C}$, dans le domaine biphasé, et on observe des pics de diffraction, dont l'intensité dépend de la longueur de cohérence $\xi$ du faisceau (dont les valeurs sont indiquées dans l'insert). Pour une longueur de cohérence inférieure ou égale à 10,3 nm, l'un des pics se dédouble (flèches), ce qui indique la présence d'inhomogénéités d'épaisseur.

(b) Interprétation des mesures. À $30^{\circ} \mathrm{C}$, la bicouche est en phase gel (schématisée par des têtes mauves et des queues jaune vif). À $42^{\circ} \mathrm{C}$ et plus, la bicouche est fluide (têtes bleu ciel et queues jaune pâle). De $38^{\circ} \mathrm{C}$ à $41^{\circ} \mathrm{C}$, on observe des inhomogénéités d'épaisseur, qu’on interprète comme une coexistence entre domaines gels et fluides. (D’après [8].)

\section{$\gg>$}

récepteurs transmembranaires. Grâce, ici encore, à la réflectivité de neutrons et à la substitution d'hydrogène par du deutérium, il a été possible d'étudier comment évolue la distribution du cholestérol quand du GM1 est incorporé dans un seul feuillet (fig. 4). Cette répartition asymétrique du GM1 favorise une répartition asymétrique du cholestérol, ce qui confirme le couplage entre ces deux molécules. Ce résultat, déjà prédit, n'avait pourtant jamais été observé.

Enfin, les microdomaines latéraux (" radeaux ") dans les membranes sont des structures dynamiques prédites mais très difficiles à observer, probablement parce qu'ils fluctuent latéralement à des échelles de temps entre la nano- et la microseconde. Des expériences de diffraction de neutrons ont permis d'apporter la première preuve expérimentale directe de la coexistence de domaines gel et fluide dans une membrane (faite d'un seul type de phospholipide), autour de sa température de transition (fig. 5) [8]. L'astuce a consisté à utiliser la diffraction de neutrons sur des empilements multilamellaires de bicouches, en faisant varier de 3 à 24 nanomètres la "longueur de cohérence " du faisceau (extension de l'onde plane au niveau de l'échantillon). Cette quantité définit la limite inférieure de la taille des structures qui peuvent être observées. Selon la valeur de cette longueur, on peut observer des pics de diffraction, qui révèlent que les lipides sont ordonnés, tant dans le plan de la membrane que perpendiculairement à ce plan. Cela permet d'estimer l'échelle des domaines : ils sont de taille nanométrique. Au cours de la transition de phase principale, on observe la coexistence de domaines en phase gel et fluide. Cette technique devrait pouvoir être étendue aux lipides extraits de membranes naturelles.

\section{Perspectives}

Les techniques dans l'espace réel, comme le microscope à force atomique, ont certes beaucoup progressé au cours des dernières décennies. Cependant, la diffusion de neutrons donne des résultats complémentaires et reste très utilisée. Une de ses applications de plus en plus fréquente est l'étude de la structure à l'échelle nanométrique de membranes modèles et de leurs interactions avec des protéines ou peptides participant à des fonctions cellulaires importantes.

La deutération de protéines devient une technique courante, qui permet d'utiliser les neutrons pour affiner des détails des interactions protéines-lipides et de la structure des protéines aux interfaces. La diffusion des neutrons aux petits angles a une résolution de l'ordre du nanomètre et, contrairement aux techniques cristallogra- phiques, peut être utilisée pour sonder une protéine dans son environnement natif, comme au sein d'une membrane ou d'un complexe avec d'autres protéines. De façon analogue, on peut aussi suivre la cinétique de changement de conformation, d'agrégation ou d'interaction de lipides.

Dans des travaux récents, nous montrons qu'il est possible de préparer des extraits de vraies membranes de levures naturelles, pour en faire des bicouches reproductibles et stables. Cela nous a permis d'étudier, par exemple, le mécanisme d'interaction d'une molécule antibiotique comme l'amphotéricine, avec des membranes naturelles contenant du stérol. En effet, des bactéries sont tuées par cette molécule, tandis que les cellules humaines lui résistent. Cela est probablement lié à la présence, dans les membranes humaines, de cholestérol qui a une structure différente de celle des stérols présents dans les bactéries. Nous avons constaté que l'amphotéricine interagit plus fortement avec les membranes de levures qui possèdent du stérol, qu'avec des membranes dépourvues de stérols ou contenant du cholestérol. Nous avons pu déterminer la structure exacte de la membrane et la localisation de la molécule antibiotique sur la membrane.

Dans la biologie de l'avenir, la physique devrait jouer un rôle majeur. Si l'on exploite pleinement leur potentiel, les neutrons pourront aider à démêler les secrets de la vie.

\section{Remerciements}

L'auteure souhaite remercier François Graner pour son travail d'édition et de correction de cet article, et pour avoir fortement contribué au début de ces travaux, il y a longtemps... sur les membranes modèles.

\section{Références}

1 0. Mouritsen, Life as a Matter of Fact: The Emerging Science of Lipidomics, Springer-Verlag (2005).

2- R. Lipowsky, "Generic interactions of flexible membranes", dans Structure and Dynamics of Membranes, Vol. A, pp. 521-602, eds R. Lipowsky et E. Sackmann, Elsevier, Amsterdam (1995).

3• H.P. Wacklin, Current Opinion In Colloid \& Interface Science, 15 (2010) 445-454.

4- A. Hemmerle et al., Proc. Natl. Acad. Sci. U. S. A., 109 (2012) 19938-19942

5• B. Jacrot, Rep. Prog. Phys., 39 (1976) 911-953.

$6 \bullet V$. Rondelli, G. Fragneto et al., Biochimica et Biophysica Acta, 1818 (2012) 2860-2867.

7• Y. Gerelli et al., Langmuir, 29 (2013) 12762-12769.

8• C. L. Armstrong et al., Soft Matter, 8 (2012) 4687-4694. 